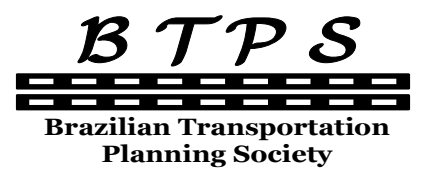

Planning Society
Journal of Transport Literature

Vol. 8, n. 4, pp. 164-186, Oct. 2014

Research Directory
JTL | RELIT

www.journal-of-transport-literature.org ISSN 2238-1031

\title{
Análise de viabilidade econômica da participação de cooperativas populares na cadeia de fornecimento de óleo residual de fritura a fábricas de biodiesel
}

[Viability analysis of popular cooperatives in the participation of residual frying oil to biodiesel plants supply chain]

\author{
Ricardo César da Silva Guabiroba, Márcio de Almeida D’Agosto, Luíza Santana Franca* \\ Federal University of Rio de Janeiro - Brazil
}

Submitted 9 Sep 2013; received in revised form 28 Jan 2014; accepted 26 Mar 2014

\begin{abstract}
Resumo
O óleo residual de fritura (ORF) é a matéria-prima mais barata para a produção de biodiesel. É uma prática de cooperativas populares a coleta e o agrupamento do ORF de modo a gerar escala de fornecimento a fábricas de biodiesel. No entanto, para a sobrevivência e atuação dessas cooperativas, estas devem gerar lucro. Assim sendo, este artigo tem como contribuição a estruturação e a apresentação de um procedimento composto por quatro etapas, voltado a auxiliar cooperativas populares quanto à análise de geração de lucro referente à operação de coleta do ORF. Esse procedimento foi aplicado à realidade de uma associação de cooperativas localizada na região metropolitana do Rio de Janeiro. A partir dessa aplicação, verificou-se que se a coleta fosse realizada de modo planejado a partir de um software de roteirização, o seu custo teria uma redução de quase $34 \%$ em relação ao custo real praticado e a associação de cooperativas estaria apta a gerar lucro, mesmo se o preço do ORF atingisse seu menor preço de mercado, estando protegida contra variações de preço estabelecidas pelo mercado.
\end{abstract}

Palavras-Chave: óleo residual de fritura, cooperativas, biodiesel, viabilidade econômica de cooperativas.

\begin{abstract}
The residual oil frying (ROF) is the cheapest raw material for biodiesel production. The collection and grouping of ROF is a popular practice of cooperatives, that's supply biodiesel plants. However, for the survival and performance of these cooperatives, these should generate profit. Therefore, this article represents a contribution by structuring and presentation a procedure of four stages, consisting in assist popular cooperatives in the analysis of generating profit by the ROF's collection and operation. This procedure was applied in an association of cooperatives located in the metropolitan region of Rio de Janeiro. From this application, it was concluded that the collection should planned by a software routing and her cost would have a reduction of almost $34 \%$ over the actual cost applied and the association of cooperatives would be able to generate profits and will be protected against market fluctuations, even if the price of ROF reached its lowest market price.
\end{abstract}

Key words: residual frying oil, cooperatives, biodiesel, economic viability of cooperatives.

*Email: luizasfranca@poli.ufrj.br.

\section{Recommended Citation}

Guabiroba, R. C. S., D'Agosto, M. A. and Franca, L. S. (2014) Análise de viabilidade econômica da participação de cooperativas populares na cadeia de fornecimento de óleo residual de fritura a fábricas de biodiesel. Journal of Transport Literature, vol. 8, n. 4, pp. 164-186.

- JTL/RELIT is a fully electronic, peer-reviewed, open access, international journal focused on emerging transport markets and published by BPTS - Brazilian Transport Planning Society. Website www.journal-of-transport-literature.org. ISSN 2238 -1031. 


\section{Introdução}

Este artigo tem como objetivo a criação de um procedimento de análise a fim de verificar se cooperativas populares são capazes de gerar lucro em cenários que variam quanto ao preço de mercado do Óleo Residual de Fritura - ORF (que define a receita) e quanto à estruturação do processo de coleta desse óleo (que define o custo). Se há lucro, é viável a participação da cooperativa no fornecimento do ORF a fábricas onde esse óleo é utilizado como matériaprima para a produção de biodiesel. O referido procedimento é composto por quatro etapas envolvendo o conhecimento do mercado de ORF, o conhecimento das operações de coleta praticadas, a análise e a definição do intervalo em que o preço de mercado do ORF pode variar para a ocorrência de lucro operacional e, por fim, a melhoria das operações de coleta.

Neste contexto, este artigo contribui para a estruturação e a apresentação de um procedimento voltado a auxiliar cooperativas populares. A sobrevivência dessas cooperativas é fundamental para que o ORF seja fornecido a fábricas de biodiesel. A partir dessa prática, essas fábricas são abastecidas por uma matéria-prima mais barata, as cooperativas populares tornam-se um meio de geração de renda para comunidades carentes e, além disso, o ORF está sendo destinado de modo adequado. O procedimento propõe ainda a utilização de um software de roteirização voltado a reduzir o custo de uma operação de coleta e destino de um resíduo.

Para a redução desse custo, é necessário reduzir a distância do percurso ou rota definida para a coleta. Nos últimos anos, alguns trabalhos têm sido publicados (Nuortio et al., 2006; Leduc et al., 2009; Araujo et al., 2010; Singh et al., 2010) sobre otimização ou aprimoramento de redes logísticas obtidos por modelos matemáticos ou heurísticos. No entanto, poucos trabalhos com essa abordagem (Ghose et al., 2006;. Alvarez et al., 2008; Tavares et al., 2009) foram publicados usando algum tipo de software de roteirização, conforme aborda este trabalho.

Assim sendo, foi possível constatar, a partir da aplicação do procedimento, que a associação de cooperativas estudada não tinha assegurada a geração de lucro em um cenário com o preço mais baixo já estipulado pelo mercado. No entanto, com a redução do custo de coleta a partir de um software de roteirização (redução de quase 34\%), a associação de cooperativas passou 
a gerar lucro independentemente da variação de preços, o que garante sua sobrevivência em um mercado instável fortemente influenciado por grandes fornecedores do ORF.

Neste sentido, com a atuação de cooperativas, coleta-se o ORF, uma matéria-prima mais barata que o óleo vegetal para a produção do biodiesel (Bhatti, et al., 2008; Issariyakul, 2008; Corro et al., 2010). Sabe-se que grande parte do custo total de produção de biodiesel equivale ao custo do óleo vegetal (70-95\%), de acordo com Zhang et al., 2003; Haas et al., 2006; Phan, 2008; Glisic et al., 2009; Sakai et al., 2009; Wen et al., 2010; Liu et al., 2010; Kiss et al., 2010. Sabe-se ainda que o alto custo do óleo vegetal é o principal fator econômico que faz com que o custo do biodiesel não seja competitivo (Predojevic, 2008; Liu et al., 2010). É estimado que o biodiesel produzido a partir do óleo virgem seja de $10 \%$ a $50 \%$ mais caro que o óleo diesel de petróleo (IEA, 2008; Banerjee e Chakraborty, 2009; Han et al., 2009).

Com base no exposto, são utilizados métodos para a elaboração deste artigo, como pesquisa bibliográfica e documental, observação e coleta de dados em campo e roteirização de veículos obtida com o auxílio de um software. Assim sendo, para o alcance do objetivo proposto, a Seção 1 apresenta revisão bibliográfica sobre a produção de biodiesel e as principais matériasprimas utilizadas no mundo. Na Seção 2, são apresentados custos de aquisição e de transporte de óleos vegetais virgens e residuais, com ênfase para a definição do custo de coleta do ORF. Já a Seção 3 apresenta a contribuição deste trabalho, que se trata do procedimento proposto. $\mathrm{Na}$ Seção 4, esse procedimento é aplicado à realidade de uma associação de cooperativas localizada na região metropolitana do Rio de Janeiro (Brasil) e são discutidos os resultados dessa aplicação. Finalmente, é apresentada a Seção de conclusões e recomendações do estudo. 


\section{Biodiesel no mundo e potenciais matérias-primas para sua produção}

As energias renováveis podem ser consideradas energias sustentáveis (Peidong et al., 2009), podendo ser potenciais candidatas para a superação dos problemas de esgotamento gradual dos combustíveis fósseis (Karabulut et al., 2010), bem como o aquecimento global causado pela emissão de gases de efeito estufa (Hwang, 2010). Algumas das mais notáveis fontes de energia renovável, capazes de substituir os combustíveis fósseis, incluem, entre outros: água, energia solar e eólica e os biocombustíveis, como biodiesel (Atadashi et al., 2011).

O biodiesel é um combustível alternativo que pode substituir o óleo diesel, largamente utilizado nos setores de transporte, agricultura, comercial, doméstico e industrial (Basha et al., 2009; Demirbas, 2009). Além disso, esse biocombustível possui propriedades semelhantes às do óleo diesel produzido a partir de petróleo e pode ser usado diretamente nos motores a diesel atuais ou usado como uma mistura com óleo diesel bruto (Wassell Jr. e Dittmer, 2006; Vicente, et al., 2007 , Helwani et al., 2009). Por esta razão, o biodiesel tem recentemente atraído atenção, em diversos países, devido à sua disponibilidade, renovabilidade, nãotoxicidade, melhor qualidade das emissões e sua biodegradabilidade (Chen et al., 2009; Demirbas, 2009; Wei Li et al., 2009; Hayyan et al., 2010; Liu et al., 2010; Patil et al., 2010).

Em geral, a produção de biodiesel no mundo tem se elevado em virtude de políticas governamentais destinadas à substituição de uma percentagem de óleo diesel por biodiesel (Marchetti e Errazu, 2008; Jegannathan et al., 2011). Em 2009, a produção global de biodiesel foi mais de 15 bilhões de litros e a previsão é chegar a mais de 40 bilhões de litros em 2019 (OECD, 2010). A Europa tem dominado a indústria do biodiesel, incentivando a produção por meio de isenções fiscais e metas nacionais estabelecidas pelas diretivas da União Européia (Apostolakou et al., 2009). Em 2009, a Europa produziu mais de 10 bilhões de litros.

A Alemanha é o país que mais produz biodiesel na Europa, correspondendo a quase $28 \%$ da produção total. Além disso, a Alemanha também possui a maior capacidade de produção em 2010 (22,5\%). Juntos, Alemanha, França, Espanha e Itália possuem 63,5\% da capacidade de produção total da União Européia (EBB, 2010). Outro produtor representativo mundial de biodiesel são os EUA, com uma capacidade anual de produção de cerca de 10 bilhões de litros. No entanto, a produção de biodiesel nos EUA é menor em relação aos países da União Européia (2,65 bilhões de litros em 2008) (NBB, 2009). Em relação ao Brasil, a capacidade de 
produção de biodiesel foi de 4,3 bilhões de litros em 2009 e sua produção real foi de 1,6 bilhões de litros no mesmo ano (ANP, 2010).

Na Europa e nos EUA, o biodiesel deriva principalmente do óleo vegetal, como óleo de soja, óleo de colza e óleo de girassol. Em alguns países do Sudeste Asiático, o óleo de palma é usado como matéria-prima (Chen et al., 2009). Além disso, Indonésia e Malásia (países do Sudeste Asiático) exportam biodiesel derivado de óleo de palma para a União Européia (UE). EUA e Brasil também exportam biodiesel para a UE, porém é feito a partir de óleo de soja (IEA, 2008). No Brasil, de acordo com a ANP (2010), a principal matéria-prima utilizada para a produção de biodiesel é o óleo de soja (80,95\% do total de matéria-prima). Outras matériasprimas com expressiva produção são a gordura animal $(13,88 \%)$ e óleo de semente de algodão $(3,57 \%)$. O uso de ORF representa apenas $0,22 \%$ do total de matéria-prima existente.

\section{Custos de aquisição e de transporte de óleos vegetais virgens e residuais}

Com o intuito de conhecer o mercado e a operação de coleta do ORF, é necessário definir os custos de aquisição e transporte de óleos vegetais virgens e residuais. Os custos de aquisição tratam-se dos preços de mercado desses óleos obtidos por meio de pesquisa documental. Por meio desse tipo de pesquisa, obteve-se ainda o custo de transporte de óleos vegetais virgens e da gordura animal. Para a definição do custo de coleta do ORF, acompanhou-se a operação de uma associação de cooperativas localizada na região metropolitana do Rio de Janeiro (Brasil).

Esse mesmo custo de coleta foi determinado considerando rotas sugeridas por um software de roteirização. Esse software com módulo de roteirização trata-se de um Sistema de Informação Geográfica (SIG). O referido sistema separa a informação em diferentes camadas temáticas e armazena-as independentemente, permitindo relacionar a informação existente por meio da posição e topologia dos objetos, com a finalidade de gerar nova informação. Neste contexto, define-se dois custos de coleta do ORF: (1) custo da operação não estruturada da associação de cooperativas e (2) custo da operação dita estruturada, com rotas sugeridas por um software. 


\subsection{Preço das matérias-primas para a produção de biodiesel no Brasil}

No Brasil, as principais matérias-primas para a produção de biodiesel são: óleo de soja, gordura animal e óleo de algodão. Nesta etapa, faz-se necessário verificar o preço de venda dessas matérias-primas. Define-se ainda o preço do ORF comercializado na região metropolitana do Rio de Janeiro. Ao comparar esses preços, é possível verificar se é verdadeira a premissa de que o ORF é a matéria-prima disponível mais barata.

\subsubsection{Preço do óleo de soja, da gordura animal e do óleo de algodão}

Segundo a ANP (2010), o preço médio de venda do óleo de soja cotado durante os doze meses de 2009 foi de $\mathrm{R} \$ 1,72 /$ litro, com desvio padrão de $\mathrm{R} \$ 0,08 /$ litro. A Tabela 1 apresenta a estrutura de custo do biodiesel produzido a partir do óleo de soja nas cinco regiões brasileiras, conforme pesquisa realizada pelo IBP/COPPE/COPPEAD (2007). Por meio da Tabela 1, é possível observar que a participação do custo do óleo de soja no custo total de biodiesel corresponde a $76 \%$ na região sudeste, onde se encontra o local abordado neste estudo. Neste contexto, considerando o preço máximo de referência do biodiesel estabelecido pelo $19^{\circ}$ leilão promovido pela ANP (2010) igual a $\mathrm{R} \$ 2,32 /$ litro, $76 \%$ desse valor equivale a $\mathrm{R} \$ 1,76 /$ litro, dentro do intervalo cotado no ano de 2009 ( $\mathrm{R} \$ 1,72 /$ litro $\pm \mathrm{R} \$ 0,08 /$ litro).

Assim sendo, considera-se o preço de venda do óleo de soja igual a $\mathrm{R} \$ 1,72 /$ litro. Esse preço não inclui a parcela do transporte de transferência do óleo de soja para a fábrica de biodiesel. Para a definição desse valor, recorre-se novamente a Tabela 1. Na região sudeste, a participação do custo de transporte do óleo de soja no custo total do biodiesel é igual a $2 \%$. Neste sentido, estima-se a parcela do transporte como $2 \%$ de $\mathrm{R} \$ 2,32 /$ litro que corresponde a $\mathrm{R} \$ 0,05 /$ litro, um baixo custo, pois o óleo de soja é transportado em escala. Para o produtor de biodiesel, o preço estimado do óleo de soja (preço do produto mais a parcela do transporte) equivale a $\mathrm{R} \$ 1,77 /$ litro ( $\mathrm{R} \$ 1,72 /$ litro + $\mathrm{R} \$ 0,05 /$ litro).

Segue-se o mesmo raciocínio para a definição do preço da gordura animal e do óleo de algodão. Segundo a ANP (2010), o preço médio de venda da gordura animal em 2009 foi de $\mathrm{R} \$ 1,28 /$ litro com desvio padrão de $\mathrm{R} \$ 0,09 /$ litro. Para o óleo de algodão, o preço médio de venda foi de $\mathrm{R} \$ 1,93 /$ litro com desvio padrão de $\mathrm{R} \$ 0,08 /$ litro. Considera-se que o valor da parcela do transporte em escala dessas matérias-primas para a fábrica de biodiesel seja o 
mesmo valor estimado da parcela do transporte em escala do óleo de soja ( $\mathrm{R} \$ 0,05 /$ litro). Assim sendo, o preço estimado (preço do produto mais a parcela do transporte) é de $\mathrm{R} \$ 1,33 /$ litro para a gordura animal e de R\$1,98/litro para o óleo de algodão.

\section{Tabela 1 - Distribuição percentual do custo do biodiesel produzido a partir do óleo de soja virgem ${ }^{1}$}

\begin{tabular}{llllllll}
\hline Região & $\mathrm{A}$ & $\mathrm{B}$ & $\mathrm{C}$ & $\mathrm{D}$ & $\mathrm{E}$ & $\mathrm{F}$ & $\begin{array}{l}\text { Total } \\
(\%)\end{array}$ \\
& $(\%)$ & $(\%)$ & $(\%)$ & $(\%)$ & $(\%)$ & $(\%)$ & $(\%)$ \\
Sul & 72 & 1 & 5 & 7 & 1 & 14 & 100 \\
Cudeste & 76 & 2 & 4 & 7 & 1 & 10 & 100 \\
Nontro-Oeste & 71 & 2 & 4 & 7 & 1 & 15 & 100 \\
Norte & 70 & 3 & 4 & 7 & 2 & 14 & 100 \\
\hline Média Brasil & 71 & 3 & 4 & 7 & 2 & 13 & 100 \\
Desvio Padrão & 3,0 & 2,8 & 1,1 & 0,9 & 1,3 & 2,7 & - \\
\hline A: Pa & 8 & 2 & 9 & 4 & 9 & 100 \\
\hline
\end{tabular}

A: Participação do custo do óleo proveniente de matéria-prima cultivável (oleaginosa);

B: Participação do custo de transporte do óleo proveniente da oleaginosa;

C: Participação do custo dos insumos: álcool e catalisador;

D: Participação do custo de produção do biodiesel (mão-de-obra e energia);

E: Participação do custo de transporte do biodiesel;

F: Participação do custo com impostos.

(-): não se aplica.

\subsubsection{Preço do ORF}

Segundo a associação de cooperativas populares, o preço mínimo de venda do ORF já estabelecido no mercado foi de $\mathrm{R}$ \$ 0,55/litro e o preço máximo alcançado foi de $\mathrm{R} \$$ 1,00/litro (esses preços incluem o frete de entrega). A variação do preço de venda do ORF depende de três fatores principais: (1) da qualidade do óleo, (2) da escassez ou excesso de oferta do ORF no mercado e (3) das negociações entre fornecedores e compradores. Os cinco principais fornecedores do ORF na região metropolitana do Rio de Janeiro são apresentados por meio da Tabela 2. A associação de cooperativas populares é o fornecedor com menor potencial de oferta, uma desvantagem para influenciar uma possível decisão de aumentar o preço de venda, principalmente por não garantir uma escala e uma regularidade de entrega. Neste contexto, é

\footnotetext{
${ }^{1}$ Fonte: IBP/COPPE/COPPEAD (2007).
} 
possível observar que o preço de venda do ORF é uma variável que depende do mercado. No cenário atual, a associação de cooperativas populares encontra-se em uma posição desfavorável, não sendo capaz de influenciar a precificação do ORF.

Tabela 2 - Concorrentes na venda de ORF na região metropolitana do Rio de Janeiro ${ }^{2}$

\begin{tabular}{lll}
\hline Concorrente & Cidade & $\begin{array}{l}\text { Quantidade coletada mensal } \\
\text { (toneladas) }\end{array}$ \\
\hline MBR & Duque de Caxias & 300 \\
Rio Óleo & Rio de Janeiro & 150 \\
Disque Óleo Vegetal Usado & Duque de Caxias & 100 \\
JW Dias Comércio & Rio de Janeiro & 80 \\
Associação de cooperativas populares & Rio de Janeiro & 40 \\
\hline
\end{tabular}

\subsection{Observação da operação de coleta realizada pela associação de cooperativas}

Para a definição do custo de coleta do ORF, foram acompanhadas cinco operações de coleta realizadas em cinco dias distintos. Durante esse acompanhamento, as seqüências de visitas e os dados de distância percorrida entre os pontos visitados foram registrados, conforme Tabela 3. Essa operação de coleta foi considerada como não estruturada, por diversas razões, como (1) definição de roteiros de coleta de forma intuitiva, (2) inexistência de um fluxo de informações, (3) desconhecimento da zona de coleta pelo motorista, (4) falta de funcionários para exercer as atividades de planejamento e de operação e (5) falta de treinamento.

Para a realização das coletas, a associação de cooperativas populares contou com dois veículos (furgões) disponibilizados pelo governo do estado do Rio de Janeiro. Como não se possuía dados históricos operacionais desses veículos, foram utilizados dados de outro veículo (também um furgão) com características semelhantes. A Tabela 4 apresenta a composição de custos fixos e variáveis desse veículo-tipo, com utilização de 2.000 quilômetros rodados mensais. $\mathrm{O}$ custo total para esse veículo-tipo é estimado em $\mathrm{R} \$ 2,685 /$ quilômetro.

\footnotetext{
${ }^{2}$ Fonte: elaboração própria a partir de PROVE (2008).
} 
Tabela 3 - Dados de distância percorrida (quilômetros) coletados em campo ${ }^{3}$

\begin{tabular}{|c|c|c|c|c|c|}
\hline Coleta & Paradas & Doador & Bairro (Cidade*) & Arco & Distância \\
\hline \multirow{8}{*}{1} & P0 & Central Alfa & Cidade Universitária (RJ) & $\mathrm{P} 0-\mathrm{P} 1$ & 11 \\
\hline & $\mathrm{P} 1$ & Restaurante & Bonsucesso (RJ) & $\mathrm{P} 1-\mathrm{P} 2$ & 11 \\
\hline & $\mathrm{P} 2$ & Cooperativa & Engenho da Rainha (RJ) & $\mathrm{P} 2-\mathrm{P} 3$ & 7 \\
\hline & $\mathrm{P} 3$ & Bar & Complexo do Alemão (RJ) & $\mathrm{P} 3-\mathrm{P} 4$ & 3 \\
\hline & $\mathrm{P} 4$ & Condomínio & Del Castilho (RJ) & $\mathrm{P} 4-\mathrm{P} 5$ & 18 \\
\hline & P5 & Escola & Quintino Bocaiúva $(\mathrm{RJ})$ & $\mathrm{P} 5-\mathrm{P} 6$ & 20 \\
\hline & P6 & Escola & Caju (RJ) & $\mathrm{P} 6-\mathrm{P} 0$ & 8 \\
\hline & \multicolumn{4}{|c|}{ Distância total percorrida (quilômetros) } & 78 \\
\hline \multirow{8}{*}{2} & P0 & Central Alfa & Cidade Universitária (RJ) & $\mathrm{P} 0-\mathrm{P} 1$ & 23 \\
\hline & $\mathrm{P} 1$ & Condomínio & Irajá (RJ) & $\mathrm{P} 1-\mathrm{P} 2$ & 11 \\
\hline & $\mathrm{P} 2$ & Cooperativa & Engenho da Rainha (RJ) & $\mathrm{P} 2-\mathrm{P} 3$ & 11 \\
\hline & $\mathrm{P} 3$ & Cooperativa & Piedade (RJ) & $\mathrm{P} 3-\mathrm{P} 4$ & 7 \\
\hline & $\mathrm{P} 4$ & Casa & Engenho Novo (RJ) & $\mathrm{P} 4-\mathrm{P} 5$ & 3 \\
\hline & P5 & Condomínio & Engenho Novo (RJ) & P5 - P6 & 9 \\
\hline & P6 & Cooperativa & Tijuca (RJ) & $\mathrm{P} 6-\mathrm{P} 0$ & 14 \\
\hline & \multicolumn{4}{|c|}{ Distância total percorrida (quilômetros) } & 78 \\
\hline \multirow{9}{*}{3} & P0 & Central Alfa & Cidade Universitária (RJ) & $\mathrm{P} 0-\mathrm{P} 1$ & 12 \\
\hline & $\mathrm{P} 1$ & Restaurante & Vila da Penha (RJ) & $\mathrm{P} 1-\mathrm{P} 2$ & 5 \\
\hline & $\mathrm{P} 2$ & Condomínio & Irajá (RJ) & $\mathrm{P} 2-\mathrm{P} 3$ & 9 \\
\hline & $\mathrm{P} 3$ & Cooperativa & Irajá (RJ) & $\mathrm{P} 3-\mathrm{P} 4$ & 11 \\
\hline & $\mathrm{P} 4$ & Igreja & Piedade (RJ) & $\mathrm{P} 4-\mathrm{P} 5$ & 2 \\
\hline & P5 & Clube & Engenho de Dentro (RJ) & $\mathrm{P} 5-\mathrm{P} 6$ & 3 \\
\hline & P6 & Cozinha industrial & Engenho Novo (RJ) & $\mathrm{P} 6-\mathrm{P} 7$ & 10 \\
\hline & P7 & Apartamento & Rio Comprido (RJ) & $\mathrm{P} 7-\mathrm{P} 0$ & 12 \\
\hline & \multicolumn{4}{|c|}{ Distância total percorrida (quilômetros) } & 64 \\
\hline \multirow{11}{*}{4} & P0 & Central Alfa & Cidade Universitária (RJ) & $\mathrm{P} 0-\mathrm{P} 1$ & 11 \\
\hline & $\mathrm{P} 1$ & Cooperativa & Brás de Pina (RJ) & $\mathrm{P} 1-\mathrm{P} 2$ & 7 \\
\hline & $\mathrm{P} 2$ & Cooperativa & Engenho da Rainha (RJ) & $\mathrm{P} 2-\mathrm{P} 3$ & 10 \\
\hline & P3 & Bar & Complexo do Alemão (RJ) & $\mathrm{P} 3-\mathrm{P} 4$ & 6 \\
\hline & $\mathrm{P} 4$ & Cooperativa & Maria da Graça $(\mathrm{RJ})$ & $\mathrm{P} 4-\mathrm{P} 5$ & 8 \\
\hline & P5 & Condomínio & Andaraí (RJ) & $\mathrm{P} 5-\mathrm{P} 6$ & 5 \\
\hline & P6 & Vila & Vila Isabel (RJ) & $\mathrm{P} 6-\mathrm{P} 7$ & 16 \\
\hline & $\mathrm{P} 7$ & Cozinha industrial & Botafogo (RJ) & $\mathrm{P} 7-\mathrm{P} 8$ & 2 \\
\hline & P8 & Buffet & Botafogo (RJ) & $\mathrm{P} 8-\mathrm{P} 9$ & 7 \\
\hline & P9 & Condomínio & Flamengo (RJ) & $\mathrm{P} 9-\mathrm{P} 0$ & 23 \\
\hline & \multicolumn{4}{|c|}{ Distância total percorrida (quilômetros) } & 95 \\
\hline \multirow{7}{*}{5} & $\mathrm{P} 0$ & Central Alfa & Cidade Universitária (RJ) & $\mathrm{P} 0-\mathrm{P} 1$ & 19 \\
\hline & $\mathrm{P} 1$ & Cooperativa & Tijuca (RJ) & $\mathrm{P} 1-\mathrm{P} 2$ & 13 \\
\hline & $\mathrm{P} 2$ & Cooperativa & Piedade (RJ) & $\mathrm{P} 2-\mathrm{P} 3$ & 10 \\
\hline & P3 & Cooperativa & Inhaúma (RJ) & $\mathrm{P} 3-\mathrm{P} 4$ & 14 \\
\hline & $\mathrm{P} 4$ & Cooperativa & Penha Circular (RJ) & $\mathrm{P} 4-\mathrm{P} 5$ & 9 \\
\hline & P5 & Cooperativa & Jardim Gramacho (DC) & $\mathrm{P} 5-\mathrm{P} 0$ & 49 \\
\hline & \multicolumn{4}{|c|}{ Distância total percorrida (quilômetros) } & 114 \\
\hline
\end{tabular}

* RJ: Rio de Janeiro; DC: Duque de Caxias.

\footnotetext{
${ }^{3}$ Fonte: elaboração própria.
} 
Tabela 4 - Custo operacional do veículo-tipo com 2.000 quilômetros rodados 4

\begin{tabular}{llll}
\hline Custo & Itens & $\mathrm{R} \$ / \mathrm{km}$ & $\%$ \\
\hline \multirow{4}{*}{ Fixo } & Depreciação operacional & 0,2565 & 9,6 \\
& Remuneração do capital & 0,3665 & 13,7 \\
& Salário do motorista & 0,7625 & 28,4 \\
& Salário do Ajudante & 0,3813 & 14,2 \\
& Licenciamento e seguro obrigatório & 0,0815 & 3,0 \\
& Seguro facultativo & 0,1810 & 6,7 \\
& Despesas administrativas & 0,1970 & 7,3 \\
\cline { 2 - 4 } & Subtotal custos fixos & 2,2263 & 82,9 \\
\hline \multirow{3}{*}{ Variável } & Combustível & 0,2875 & 10,7 \\
& Pneus, câmaras e recapagens & 0,0397 & 1,5 \\
& Manutenção (peças e mão-de-obra) & 0,0781 & 2,9 \\
& Lubrificante do motor & 0,0128 & 0,5 \\
& Lubrificante da transmissão & 0,0006 & 0,0 \\
& Lavagem e lubrificação & 0,0400 & 1,5 \\
\cline { 2 - 4 } & Subtotal custos variáveis & 0,4587 & 17,1 \\
\hline Custo Total & & 2,6850 & 100,0 \\
\hline
\end{tabular}

\subsection{Redução da distância percorrida durante as operações de coleta}

Após a observação e a coleta de dados em campo, definiu-se o tipo de problema a ser solucionado. A operação tem início com a partida do veículo que deve visitar, apenas uma vez, todos os pontos indicados para a coleta. Após as visitas, o motorista deve retornar para o ponto de parada inicial (P0), onde o ORF é armazenado e tratado. O objetivo é minimizar as distâncias totais percorridas. Além disso, o volume total coletado em cada rota deve ser menor ou igual à capacidade do veículo. Esse problema é conhecido como o Problema de Roteirização de Veículos (PRV). A estrutura de rede de pontos visitada é chamada de grafo $G=(V, E)$, onde $V=\left\{u 0, u_{1}, \ldots, u_{n}\right\}$ é o conjunto de vértices e $E=\left\{\left(u_{i}, u_{j}\right): u_{i}, u_{j} \in V\right\}$ é o conjunto de arcos (Tarantilis, 2005), sendo u( $0,1, n)$ os vértices ou pontos visitados.

\footnotetext{
${ }^{4}$ Fonte: adaptado de Transporte Moderno (2008). 
Outras características desse PRV são apresentadas a partir da Tabela 5. Essas características foram classificadas de acordo com os componentes de um sistema de transportes: via, veículo, terminal e controle. Considerou-se que as vias da região metropolitana do Rio de Janeiro compõem as arestas de um grafo direcionado com a consideração necessária das mãos de direção. Como a associação de cooperativas contou com dois veículos disponibilizados pelo governo do estado do Rio de Janeiro, considerou-se uma frota homogênea sempre disponível em horário comercial. Além disso, o PRV tratado possui apenas uma central (P0) e os pontos de parada também podem ser considerados um nó do grafo direcionado. Quanto ao controle do sistema de coleta, este deve considerar a troca de recipientes cheios por vazios nos pontos de parada, a princípio sem restrição de programação. Considerou-se ainda que o volume de um pedido nunca será maior que a capacidade do veículo e que cada pedido poderá ser atendido em apenas um dia. Além disso, durante a operação, mais pedidos não são incluídos (planejamento estático) e a natureza do pedido de coleta pode ser facilmente conhecida.

\section{Tabela 5 - Outras características do PRV observadas durante as cinco operações de coleta ${ }^{5}$}

\begin{tabular}{|c|c|c|}
\hline Sistema de transportes & Item de caracterização & Característica \\
\hline Via & Tipo de grafo & Direcionado \\
\hline \multirow{3}{*}{ Veículo } & Características físicas & Frota homogênea \\
\hline & & capacidade) \\
\hline & Disponibilidade de veículos & Horário comercial \\
\hline \multirow{2}{*}{$\begin{array}{l}\text { Terminal (pontos de } \\
\text { parada) }\end{array}$} & Localização & Nós \\
\hline & Número de centrais (P0) & Única \\
\hline \multirow{6}{*}{ Controle } & Restrição de programação & Não há \\
\hline & Pedido & Coleta \\
\hline & Volume do pedido & Menor que a capacidade do veículo \\
\hline & Natureza do pedido & Determinística \\
\hline & Necessidade de atendimento & 1 dia \\
\hline & Planejamento & Estático \\
\hline
\end{tabular}

\footnotetext{
${ }^{5}$ Fonte: elaboração própria.
} 
A partir da determinação do tipo de problema e de suas características, o próximo passo tratou-se da definição do software a ser utilizado para roteirizar as cinco operações de coleta acompanhadas em campo. O software escolhido foi o TransCAD®, muito utilizado no Brasil e disponível para uso em ambiente acadêmico através de licenças pagas. Esse software é um Sistema de Informação Geográfica (SIG) que possui vários módulos, dentre eles módulos para a resolução de problemas de sistemas de transporte. $\mathrm{O}$ TransCAD ${ }^{\circledR}$ possui ferramentas para o cálculo de demandas de viagens, criação de modelos de geração de viagens, cálculo do caminho mínimo, resolução do problema de transporte, localização de instalações, problema do caixeiro viajante, que visa minimizar os custos de uma rota, e do carteiro chinês, que busca o caminho mais curto ou circuito fechado, além do problema de roteirização de veículos.

Antes da realização do processo de roteirização no software, verificou-se a base viária da região metropolitana do Rio de Janeiro (camada de linhas representando as vias urbanas) disponível em meio acadêmico. Ao verificar a base viária, notou-se que faltavam algumas vias e as vias existentes estavam sem as informações de mão de direção nas zonas de tráfego onde se realizaram as coletas. Neste sentido, fez-se necessário inserir todas essas informações de modo a tornar consistentes os resultados da roteirização. Após a melhoria da base viária, o software foi programado para realizar a roteirização das cinco operações de coleta (uma por vez). Os resultados da roteirização são rotas de visitas aos pontos de parada e a trajetória que deve ser percorrida pelo veículo. Considerados como resultados de uma operação de coleta estruturada, esses dados são apresentados por meio da Tabela 6.

Após a obtenção dos resultados da roteirização de veículos, fez-se necessário verificar sua consistência. Para essa verificação, optou-se por comparar os valores de distância dos arcos (P0-P1, P1-P2, P2-P3, P3-P4, P4-P5, P5-P6, P6-P0, por exemplo, para a primeira coleta), obtidos por meio do software TransCAD® e de três softwares de caminho mínimo (Apontador, MapLink e MapasTerra) que podem ser acessados livremente por meio da internet. $\mathrm{O}$ parâmetro de comparação foi definido como a média das distâncias dos arcos obtidas pelos três softwares encontrados na internet. Em cada uma das operações de coleta, observou-se a existência de proximidade entre as distâncias obtidas pelo software TransCAD® e as médias das distâncias obtidas pelos três softwares consultados na internet (desvio médio encontrado de 6\%). Neste sentido, constatou-se a consistência dos resultados obtidos pelo software TransCAD® após a realização da melhoria da base viária. 
Tabela 6 - Distância percorrida (quilômetros) obtida por meio do software TransCAD

\begin{tabular}{|c|c|c|c|c|c|}
\hline Coleta & Paradas & Doador & Bairro (Cidade*) & Arco & Distância \\
\hline \multirow{8}{*}{1} & P0 & Central Alfa & Cidade Universitária (RJ) & $\mathrm{P} 0-\mathrm{P} 1$ & 4,3 \\
\hline & P1 & Restaurante & Bonsucesso (RJ) & $\mathrm{P} 1-\mathrm{P} 6$ & 8,5 \\
\hline & P6 & Escola & Caju (RJ) & $\mathrm{P} 6-\mathrm{P} 4$ & 5,6 \\
\hline & P4 & Condomínio & Del Castilho (RJ) & $\mathrm{P} 4-\mathrm{P} 5$ & 7,6 \\
\hline & P5 & Escola & Quintino Bocaiúva (RJ) & $\mathrm{P} 5-\mathrm{P} 2$ & 5,6 \\
\hline & $\mathrm{P} 2$ & Cooperativa & Engenho da Rainha (RJ) & $\mathrm{P} 2-\mathrm{P} 3$ & 5,0 \\
\hline & $\mathrm{P} 3$ & Bar & Complexo do Alemão (RJ) & $\mathrm{P} 3-\mathrm{P} 0$ & 6,4 \\
\hline & \multicolumn{4}{|c|}{ Distância mínima total a ser percorrida (quilômetros) } & 43,0 \\
\hline \multirow{8}{*}{2} & P0 & Central Alfa & Cidade Universitária (RJ) & $\mathrm{P} 0-\mathrm{P} 1$ & 12,9 \\
\hline & $\mathrm{P} 1$ & Condomínio & Irajá (RJ) & $\mathrm{P} 1-\mathrm{P} 2$ & 9,0 \\
\hline & $\mathrm{P} 2$ & Cooperativa & Engenho da Rainha (RJ) & $\mathrm{P} 2-\mathrm{P} 3$ & 4,6 \\
\hline & P3 & Cooperativa & Piedade (RJ) & $\mathrm{P} 3-\mathrm{P} 4$ & 6,0 \\
\hline & P4 & Casa & Engenho Novo (RJ) & $\mathrm{P} 4-\mathrm{P} 5$ & 0,8 \\
\hline & P5 & Condomínio & Engenho Novo (RJ) & $\mathrm{P} 5-\mathrm{P} 6$ & 7,8 \\
\hline & P6 & Cooperativa & Tijuca (RJ) & $\mathrm{P} 6-\mathrm{P} 0$ & 13,2 \\
\hline & \multicolumn{4}{|c|}{ Distância mínima total a ser percorrida (quilômetros) } & 54,2 \\
\hline \multirow{9}{*}{3} & P0 & Central Alfa & Cidade Universitária (RJ) & $\mathrm{P} 0-\mathrm{P} 1$ & 9,6 \\
\hline & $\mathrm{P} 1$ & Restaurante & Vila da Penha (RJ) & $\mathrm{P} 1-\mathrm{P} 2$ & 5,0 \\
\hline & $\mathrm{P} 2$ & Condomínio & Irajá (RJ) & $\mathrm{P} 2-\mathrm{P} 3$ & 4,2 \\
\hline & P3 & Cooperativa & Irajá (RJ) & $\mathrm{P} 3-\mathrm{P} 4$ & 9,2 \\
\hline & P4 & Igreja & Piedade (RJ) & $\mathrm{P} 4-\mathrm{P} 5$ & 1,7 \\
\hline & P5 & Clube & Engenho de Dentro (RJ) & $\mathrm{P} 5-\mathrm{P} 6$ & 3,5 \\
\hline & P6 & Cozinha industrial & Engenho Novo (RJ) & $\mathrm{P} 6-\mathrm{P} 7$ & 8,0 \\
\hline & P7 & Apartamento & Rio Comprido (RJ) & $\mathrm{P} 7-\mathrm{P} 0$ & 11,0 \\
\hline & \multicolumn{4}{|c|}{ Distância mínima total a ser percorrida (quilômetros) } & 52,2 \\
\hline \multirow{11}{*}{4} & P0 & Central Alfa & Cidade Universitária (RJ) & $\mathrm{P} 0-\mathrm{P} 1$ & 9,1 \\
\hline & $\mathrm{P} 1$ & Cooperativa & Brás de Pina $(\mathrm{RJ})$ & $\mathrm{P} 1-\mathrm{P} 2$ & 5,8 \\
\hline & $\mathrm{P} 2$ & Cooperativa & Engenho da Rainha (RJ) & $\mathrm{P} 2-\mathrm{P} 3$ & 5,0 \\
\hline & P3 & Bar & Complexo do Alemão (RJ) & $\mathrm{P} 3-\mathrm{P} 4$ & 5,0 \\
\hline & P4 & Cooperativa & Maria da Graça (RJ) & $\mathrm{P} 4-\mathrm{P} 6$ & 5,3 \\
\hline & P6 & Vila & Vila Isabel (RJ) & $\mathrm{P} 6-\mathrm{P} 5$ & 2,1 \\
\hline & P5 & Condomínio & Andaraí (RJ) & $\mathrm{P} 5-\mathrm{P} 7$ & 11,7 \\
\hline & P7 & Cozinha industrial & Botafogo (RJ) & $\mathrm{P} 7-\mathrm{P} 8$ & 1,4 \\
\hline & P8 & Buffet & Botafogo (RJ) & P8 - P9 & 4,1 \\
\hline & P9 & Condomínio & Flamengo (RJ) & $\mathrm{P} 9-\mathrm{P} 0$ & 16,1 \\
\hline & \multicolumn{4}{|c|}{ Distância mínima total a ser percorrida (quilômetros) } & 65,6 \\
\hline \multirow{7}{*}{5} & $\mathrm{P} 0$ & Central Alfa & Cidade Universitária (RJ) & $\mathrm{P} 0-\mathrm{P} 4$ & 7,5 \\
\hline & P4 & Cooperativa & Penha Circular (RJ) & $\mathrm{P} 4-\mathrm{P} 5$ & 8,8 \\
\hline & P5 & Cooperativa & Jardim Gramacho (DC) & $\mathrm{P} 5-\mathrm{P} 1$ & 24,6 \\
\hline & $\mathrm{P} 1$ & Cooperativa & Tijuca (RJ) & $\mathrm{P} 1-\mathrm{P} 2$ & 10,8 \\
\hline & $\mathrm{P} 2$ & Cooperativa & Piedade (RJ) & $\mathrm{P} 2-\mathrm{P} 3$ & 6,3 \\
\hline & P3 & Cooperativa & Inhaúma (RJ) & $\mathrm{P} 3-\mathrm{P} 0$ & 6,1 \\
\hline & \multicolumn{4}{|c|}{ Distância mínima total a ser percorrida (quilômetros) } & 64,0 \\
\hline
\end{tabular}

* RJ: Rio de Janeiro; DC: Duque de Caxias. 


\subsection{Custo de transporte do ORF}

A Tabela 7 apresenta as distâncias totais reais percorridas pelo veículo durante cada uma das cinco operações de coleta. Apresenta ainda as distâncias totais reduzidas obtidas por meio do software TransCAD®. Com essas distâncias e com o custo de transporte total (R\$ 2,685/quilômetro) obtido por meio da Tabela 4, torna-se possível a realização do cálculo do custo de cada operação de coleta. Esse custo total (R\$) e a informação obtida em campo do volume coletado (litro) em cada operação de coleta permitem o cálculo do custo em R $\$ /$ litro. A partir desse cálculo, é possível obter a média do custo em $\mathrm{R} \$ /$ litro das cinco operações de coleta realizadas pela associação de cooperativas populares. Esse custo é de R \$0,68/litro para a operação atual de coleta (observada em campo, realizada de modo não estruturado e sem a existência de planejamento). Por outro lado, estima-se que esse custo seja de R $\$ 0,45 /$ litro em operações ideais de coleta (operações otimizadas, estruturadas e planejadas).

\section{Tabela 7 - Custo de cada operação de coleta realizada pela associação de cooperativas populares}

\begin{tabular}{|c|c|c|c|c|c|c|c|}
\hline \multirow[b]{2}{*}{ Coleta } & \multicolumn{2}{|c|}{ Distância (km) } & \multicolumn{2}{|c|}{ Custo (R\$) } & \multirow{2}{*}{$\begin{array}{l}\text { Volume } \\
\text { coletado } \\
\text { (litro) }\end{array}$} & \multicolumn{2}{|c|}{ Custo (R $\$ /$ litro) } \\
\hline & Atual & TransCAD® & Atual & TransCAD® & & Atual & TransCAD® \\
\hline 1 & 78,0 & 43,0 & 209,43 & 115,40 & 285 & 0,73 & 0,40 \\
\hline 2 & 78,0 & 54,2 & 209,43 & 145,61 & 885 & 0,24 & 0,16 \\
\hline 3 & 64,0 & 52,2 & 171,84 & 140,08 & 190 & 0,90 & 0,74 \\
\hline 4 & 95,0 & 65,6 & 255,08 & 176,08 & 405 & 0,63 & 0,43 \\
\hline 5 & 114,0 & 64,0 & 306,09 & 171,79 & 350 & 0,87 & 0,49 \\
\hline Média & 85,8 & 55,8 & 230,37 & 149,79 & 423 & 0,68 & 0,45 \\
\hline
\end{tabular}




\section{Procedimento de análise de geração de lucro por cooperativas populares}

Nesta seção, sugere-se um procedimento para avaliação da existência de lucro ou de prejuízo no processo de coleta realizado por cooperativas populares de ORF. Para essa avaliação, fazse necessário analisar o custo da operação de coleta e a receita de venda do ORF. Além disso, a cooperativa popular deve buscar a melhoria de seus processos de coleta de modo a reduzir o custo de operação. Neste contexto, sugere-se um procedimento composto por quatro passos básicos:

- $1^{\circ}$ passo: conhecimento do mercado de ORF (define-se o preço de venda);

- $2^{\circ}$ passo: conhecimento das operações de coleta (define-se o custo operacional);

- $3^{\circ}$ passo: análise e definição do intervalo em que o preço de mercado do ORF pode variar para a ocorrência de lucro operacional;

- $4^{\circ}$ passo: melhoria das operações de coleta (reduz-se o custo operacional).

Conhecer o mercado é determinar o preço de venda do ORF e o preço de outras matériasprimas concorrentes para a produção de biodiesel no local em estudo. Parte-se da premissa de que esses preços não são variáveis de controle das cooperativas populares, isto é, esses preços são determinados por fatores intrínsecos ao mercado. Da mesma maneira, é necessário que a cooperativa popular conheça suas operações de coleta de modo a identificar seus problemas. Deve-se registrar os dados das operações, como a distância percorrida e os tempos de serviço e de trajeto para que a efetividade do processo seja avaliada.

Em seguida, deve-se definir e analisar, por meio da Figura 1, o intervalo em que o preço de mercado do ORF pode variar para a ocorrência de lucro operacional. Essa figura apresenta uma série de retas de isocustos, cada uma representando um custo operacional que define o nível de estruturação de uma cooperativa popular. O eixo das abscissas representa o preço do ORF que é estabelecido pelo mercado e o eixo das ordenadas apresenta a margem de lucro ou de prejuízo unitário. Situa-se ainda, na Figura 1, o preço de outras matérias-primas concorrentes para a produção de biodiesel (PCX, PCY e PCZ). 


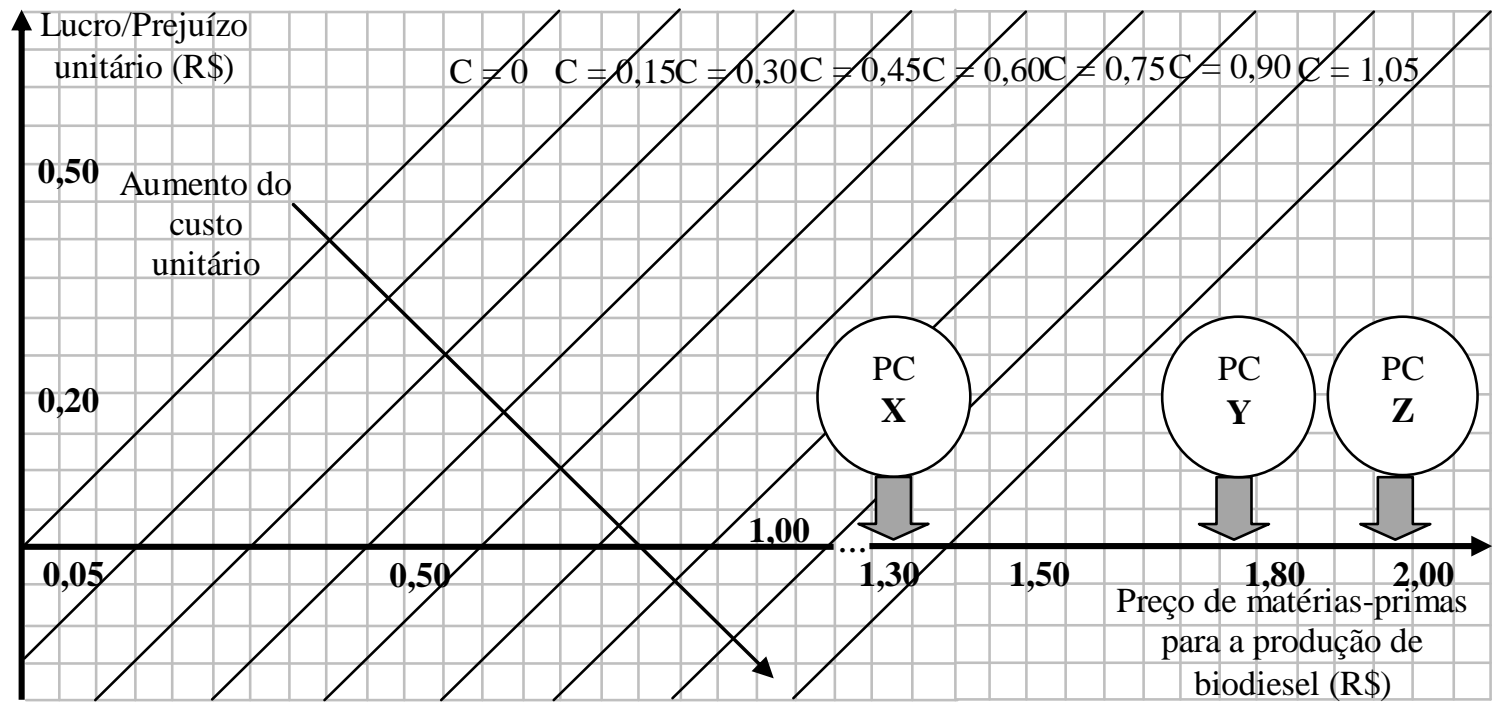

$\mathrm{C}=$ custo unitário operacional ( $\mathrm{R} \$$ por litro).

$\mathrm{PC}=$ preço da matéria-prima concorrente.

Figura 1 - Retas de isocustos de operações de coleta de ORF

Por meio da Figura 1, é possível observar que quanto menor o custo operacional, maior é o intervalo entre o ponto de lucro zero (onde a reta corta o eixo das abscissas) e o ponto que representa o preço da matéria-prima mais concorrente (PCX). Esse limite superior estabelece ainda um parâmetro para o produtor de biodiesel. Se o preço do ORF for menor que esse valor, há vantagem em adquiri-la. Neste contexto, quanto maior o intervalo de variação do preço de mercado do ORF para a ocorrência de lucro operacional, mais preparada está a cooperativa popular para sobreviver ou gerar lucro em um mercado instável onde há grande variação do preço do ORF. Por essa razão, a cooperativa popular deve buscar oportunidades de melhoria capazes de reduzir o custo operacional com o intuito de diminuir a vulnerabilidade a variações de preço de mercado do ORF que podem gerar prejuízo.

\section{Aplicação do procedimento e discussão dos resultados}

O primeiro passo do procedimento é o conhecimento do mercado. No Brasil e na região metropolitana do Rio de Janeiro, as principais matérias-primas concorrentes com o ORF são a gordura animal, o óleo de soja e o óleo de algodão. O preço estimado do óleo de algodão é de $\mathrm{R} \$ 1,98 /$ litro e do óleo de soja é de $\mathrm{R} \$ 1,77 /$ litro nessa região. A gordura animal, um tipo de matéria-prima residual como o ORF, tem preço estimado em $\mathrm{R} \$ 1,33 /$ litro (conforme item 2.1.1). O preço máximo do $\mathrm{ORF}$ estabelecido nessa região foi de $\mathrm{R} \$ 1,00 /$ litro e o preço 
mínimo foi de $\mathrm{R} \$ 0,55 /$ litro, conforme informação da associação de cooperativas populares (conforme item 2.1.2).

O segundo passo do procedimento trata-se do conhecimento das operações de coleta da associação de cooperativas populares. No processo de acompanhamento, vários problemas foram identificados, indicando que a operação real é realizada de modo não estruturado. Após o registro de dados em campo, como o trajeto e o roteiro escolhido pelo motorista, foi possível efetuar o cálculo do custo da operação real. O valor encontrado foi de R $\$ 0,68 /$ litro (conforme item 2.4).

O terceiro passo do procedimento tem como objetivo a definição e a análise do intervalo de variação do preço de mercado do ORF para a ocorrência de lucro na operação atual. Para auxiliar essa etapa, recorre-se a Figura 1. Primeiramente, deve-se identificar a reta de isocusto $\mathrm{R} \$ 0,68 /$ litro. Para essa reta ou para esse nível de estruturação, o intervalo de variação é definido pelo:

- Limite inferior: preço do ORF que zera o lucro unitário - $\mathrm{R} \$ 0,68 /$ litro;

- Limite superior: preço da matéria-prima mais concorrente com o preço do ORF para a produção de biodiesel (gordura animal) - R \$1,33/litro.

Neste contexto, quando o preço do ORF estabelecido pelo mercado é de $\mathrm{R} \$ 1,00 /$ litro (dentro do intervalo definido), observa-se que há lucro unitário igual a $\mathrm{R} \$ 0,32 /$ litro. Quando esse preço atinge um valor mínimo de $\mathrm{R} \$ 0,55 /$ litro (fora do intervalo definido), é possível verificar que não há ocorrência de lucro. Neste caso, o prejuízo unitário chega a ser de R\$0,13/litro. Neste sentido, para esse custo operacional, conclui-se que a associação de cooperativas populares não está protegida contra variações de preço do ORF no mercado em estudo. Portanto, segue-se o procedimento com a realização do quarto passo que se trata da melhoria das operações de coleta.

Entende-se como melhoria de processo a solução dos problemas observados durante o acompanhamento das operações de coleta. Inclui-se ainda a realização de um processo estruturado de planejamento com o auxílio de um software de roteirização de veículos. Por meio desse software, foi possível estimar qual seria o novo custo de operação, alcançado após essas melhorias. Esse valor foi calculado, encontrando-se o custo de R $\$ 0,45 /$ litro (conforme 
item 2.4). Nota-se ainda que tanto o custo ideal quanto o custo atual de operação são compostos apenas pelo custo de transporte, pois o ORF coletado é doado.

Para a definição e a análise do intervalo de variação do preço de mercado do ORF para o novo custo da operação estruturada, planejada e otimizada, recorre-se novamente a Figura 1 e encontra-se a reta de isocusto $\mathrm{R} \$ 0,45 /$ litro. Para essa reta ou para esse nível de estruturação, o intervalo é composto pelo:

- Limite inferior: preço do ORF que zera o lucro unitário - $\mathrm{R} \$ 0,45 /$ litro;

- Limite superior: preço da matéria-prima mais concorrente com o preço do ORF para a produção de biodiesel (gordura animal) - R \$1,33/litro.

Nesta situação, se o preço do ORF é de $\mathrm{R} \$ 1,00 /$ litro (dentro do novo intervalo definido), há a ocorrência de lucro unitário igual a R \$0,55/litro. Em um cenário menos favorável, com o preço do ORF alcançando $\mathrm{R} \$ 0,55 /$ litro (também dentro do intervalo), também há a ocorrência de lucro unitário igual a $\mathrm{R} \$ 0,10 /$ litro. Assim sendo, a redução do custo operacional de $\mathrm{R} \$ 0,68 /$ litro para $\mathrm{R} \$ 0,45 /$ litro permitiu à associação de cooperativas populares gerar lucro não apenas em um cenário favorável, mas também em um cenário desfavorável onde o preço do ORF é de R\$0,55/litro. Neste contexto, com o novo custo operacional, a associação está protegida contra variações de preço do ORF que ocorrem nesse mercado, gerando lucro e garantindo sua sobrevivência.

Quanto às matérias-primas concorrentes com o ORF para a produção de biodiesel, conforme Figura 1, tem-se:

- PCX: R \$1,33/litro para a gordura animal;

- $\quad$ PCY: R\$1,77/litro para o óleo de soja;

- PCZ: R\$1,98/litro para o óleo de algodão.

Como o preço máximo do ORF foi de $\mathrm{R} \$ 1,00 /$ litro, para este caso, é vantajoso para o produtor de biodiesel adquirir esse resíduo, isto é, pode ser vantajoso tanto para o produtor de biodiesel demandar o ORF quanto para a cooperativa popular participar da cadeia de suprimento de fábricas de biodiesel. 


\section{Conclusão}

O objetivo deste artigo tratou-se da criação de um procedimento de análise a fim de verificar se a cooperativa popular é capaz de gerar lucro em cenários que variam quanto ao preço de mercado (define a receita) e quanto à estruturação do processo de coleta (define o custo). Sendo capaz de gerar lucro, a cooperativa popular garante sua sobrevivência (e a consequente inserção de catadores) e está apta a compor a cadeia de suprimento de fábricas de biodiesel ao ofertar o ORF, uma matéria-prima gerada dispersamente em áreas urbanas.

Neste sentido, o procedimento proposto contribui para auxiliar cooperativas populares quanto à análise de geração de lucro referente à operação de coleta do ORF. O referido procedimento foi aplicado com base na realidade da operação de coleta de uma associação de cooperativas populares localizada na região metropolitana do Rio de Janeiro. A partir do melhor roteiro sugerido por um software, foi possível verificar as perdas provenientes da operação de coleta atual observada. Essas perdas foram justificadas por razões, como falta de planejamento, de treinamento, de informação e de participação dos envolvidos. Com o planejamento realizado por meio do software e com a resolução desses problemas apresentados, estimou-se a redução do custo de coleta de $\mathrm{R} \$ 0,68 / 1$ para $\mathrm{R} \$ 0,45 / 1$ (redução de quase $34 \%$ ).

Vale ressaltar que essa redução é de extrema importância para a obtenção de lucro, uma vez que o preço de mercado do ORF não é uma variável controlada pela associação de cooperativas populares. Para essa redução, um procedimento de quatro passos foi sugerido: (1) conhecimento do mercado que se trata da análise do ambiente externo à cooperativa; (2) conhecimento das operações de coleta ou dos processos internos à cooperativa; (3) conhecimento dos limites em que o preço do ORF definido pelo mercado pode variar, para ainda assim possibilitar a geração de lucro; e (4) melhoria das operações de coleta ao estruturar e planejar os processos logísticos intrínsecos à cooperativa.

A aplicação do procedimento permitiu verificar ainda que a associação de cooperativas está vulnerável a variações de preço de mercado do ORF, quando sua operação é realizada da forma como foi observada (operação atual não estruturada com custo igual a R\$0,68/1). Em um cenário desfavorável que apresenta preço de mercado igual a $\mathrm{R} \$ 0,55 / 1$, há a ocorrência de prejuízo, inviabilizando a existência dessas cooperativas voltadas a coletar o ORF com destino a fábricas de biodiesel. Após estimar o novo custo de operação, igual a $\mathrm{R} \$ 0,45 / 1$, 
obtido após o quarto passo do procedimento, verificou-se que a associação de cooperativas está preparada para gerar lucro, mesmo no cenário desfavorável mencionado (preço de mercado igual a $\mathrm{R} \$ 0,55 / 1)$, isto é, sua sobrevivência está mais bem assegurada quando a operação logística é realizada de modo estruturado.

Entende-se por estruturação das operações logísticas a abordagem sistêmica que envolve a melhoria da gestão de funções de processamento de pedidos, de transporte e de estoque. As limitações para o alcance dessa melhoria proposta no procedimento incluem a redução do esforço administrativo das cooperativas ao longo do tempo para atuar individualmente ou em conjunto. Apesar dos princípios básicos de uma cooperativa retratarem a divisão igualitária do trabalho, observou-se, no caso em estudo, divisão desigual, o que causou atritos, contribuindo para prejudicar o desempenho administrativo da associação de cooperativas.

Outra limitação envolve a obtenção e manipulação do software de roteirização pelos membros da cooperativa. Para a obtenção do software, há a limitação de recursos. Para a manipulação deste, não recebem orientação ou treinamento. Neste sentido, é necessário que essas cooperativas recebam apoio, principalmente do governo local e de universidades. Quanto à disponibilização do software, muitos centros de estudos em transporte dessas universidades e governos utilizam este tipo de ferramenta. Assim sendo, licenças poderiam ser estendidas a cooperativas. Além disso, poderiam contribuir com o treinamento para a utilização do software. Caso a cooperativa não apresente cooperados com a habilidade para manipular a ferramenta (havia no caso abordado neste trabalho), haveria ainda a opção de o governo local disponibilizar um profissional para atuar neste auxílio, pelo menos em tempo parcial.

Para estudos futuros, recomenda-se a aplicação do procedimento a outros casos de cooperativas populares que levem em consideração outras restrições impostas ao PRV tratado neste artigo. Essas restrições podem contemplar janelas de tempo, múltiplos depósitos, entrega particionada, planejamento dinâmico de coleta, entre outros. Para a estruturação e para a melhoria das operações de coleta, recomenda-se utilizar outros recursos encontrados no software TransCAD®, como a localização de terminais intermediários. 


\section{Referências}

Alvarez, J. V. L., Larrucea, M. A., Quero, S. F. C. e Valle, A. J. (2008) Optimizing the collection of used paper from small businesses through GIS techniques: The Leganés case (Madrid, Spain). Waste Management, vol. 28, pp. 282-293.

Agência Nacional do Petróleo, Gás Natural e Biocombustíveis - ANP (2010) Resumo 19 leilões ANP. p. 4, Novembro, 2010. Rio de Janeiro: ANP. Disponível em www.anp.gov.br.

Agência Nacional do Petróleo, Gás Natural e Biocombustíveis - ANP (2010) Boletim mensal do biodiesel, pp. 8-9, Setembro, 2010. Rio de Janeiro: ANP. Disponível em www.anp.gov.br.

Apostolakou, A. A., Kookos, I. K., Marazioti, C. e Angelopoulos, K. C. (2009) Techno-economic analysis of a biodiesel production process from vegetable oils. Fuel Processing Technology, vol. 90, pp. 1023-1031.

Araujo, V. K. W. S., Hamacher, S. e Scavarda, L. F. (2010) Economic assessment of biodiesel production from waste frying oils. Bioresource Technology, vol. 101, pp. 4415-4422.

Atadashi, I. M., Aroua, M. K. e Aziz, A. A. (2011) Biodiesel separation and purification: A review. Renewable Energy, vol. 36, pp. 437-443.

Banerjee, A. e Chakraborty, R. (2009) Parametric sensitivity in transesterification of waste cooking oil for biodiesel production - A review. Resources, Conservation and Recycling, vol. 53, pp. 490497.

Basha, S. A., Gopal, K. R. e Jebaraj, S. (2009) A review on biodiesel production, combustion, emissions and performance. Renewable and Sustainable Energy Reviews, vol. 13, pp. 16281634.

Bhatti, H. N., Hanif, M. A., Qasim, M. e Ata-ur-Rehman (2008) Biodiesel production from waste tallow. Fuel, vol. 87, pp. 2961-2966.

Chen, Y., Xiao, B., Chang, J., Fu, Y., Lv, P. e Wang, X. (2009) Synthesis of biodiesel from waste cooking oil using immobilized lipase in fixed bed reactor. Energy Conversion and Management, vol. 50, pp. 668-673.

Corro, G., Tellez, N., Jimenez, T., Tapia, A., Banuelos, F. e Vazquez-Cuchillo , O. (2010) Biodiesel from waste frying oil. Two step process using acidified $\mathrm{SiO} 2$ for esterification step. Catalysis Today, vol. 166, pp. 116-122.

Demirbas, A. (2009) Biodiesel from waste cooking oil via base-catalytic and supercritical methanol transesterification. Energy Conversion and Management, vol. 50, pp. 923-927.

European Biodiesel Board - EBB (2010) European Biodiesel Board Statistics, Brussels.

Ghose, M. K., Dikshit, A. K. e Sharma, S. K. (2006). A GIS based transportation model for solid waste disposal - A case study on Asansol municipality. Waste Management, vol. 26, pp. 12871293.

Glisic, S., Lukic, I. e Skala, D. (2009) Biodiesel synthesis at high pressure and temperature: Analysis of energy consumption on industrial scale. Bioresource Technology, vol. 100, pp. 6347-6354.

Haas, M. J., McAloon, A. J., Yee, W. C. e Foglia, T. A. (2006) A process model to estimate biodiesel production costs. Bioresource Technology, vol. 97, pp. 671-678.

Han, M., Yi, W., Wu, Q., Liu, Y., Hong, Y. e Wang, D. (2009) Preparation of biodiesel from waste oils catalyzed by a Brønsted acidic ionic liquid. Bioresource Technology, vol. 100, pp. 23082310 . 
Hayyan, M., Mjalli, F. S., Hashim, M. A. e Alnashef, I. M. (2010) A novel technique for separating glycerine from palm oil-based biodiesel using ionic liquids. Fuel Processing Technology, vol. 91, pp. 116-120.

Helwani, Z., Othman, M. R., Aziz, N., Fernando, W. J. N. e Kim, J. (2009) Technologies for production of biodiesel focusing on green catalytic techniques: A review. Fuel Processing Technology, vol. 90, pp. 1502-1514.

Hwang, J. J. (2010) Promotional policy for renewable energy development in Taiwan. Renewable and Sustainable Energy Reviews, vol. 14, pp. 1079-1087.

IBP - Instituto Brasileiro de Petróleo, Gás Natural e Biocombustíveis/COPPE/COPPEAD (2007) Projeto: Aspectos Técnicos e Logísticos para a Produção de Biodiesel no Brasil. Rio de Janeiro, Brasil.

International Energy Agency - IEA (2008) World Energy Outlook. Part A: Global Energy Trends to 2030 - Chapter 7: Renewable Energy Outlook, p. 173, 174 and 175.

Issariyakul, T., Kulkarni, M. G., Meher, L. C., Dalai, A. K. e Bakhshi, N. N. (2008) Biodiesel production from mixtures of canola oil and used cooking oil. Chemical Engineering Journal, vol. 140, pp. 77-85.

Jegannathan, K. R., Eng-Seng, C. e Ravindra, P. (2011) Economic assessment of biodiesel production: Comparison of alkali and biocatalyst processes. Renewable and Sustainable Energy Reviews, vol. 15, pp. 745-751.

Karabulut, A., Gedik, E., Keçebas, A. e Alkan, M. A. (2010) An investigation on renewable energy education at the university level in Turkey. Renewable Energy, vol. 36, pp. 1293-1397.

Kiss, F. E., Jovanović, M. e Bošković, G. C. (2010) Economic and ecological aspects of biodiesel production over homogeneous and heterogeneous catalysts. Fuel Processing Technology, vol. 91, pp. 1316-1320.

Leduc, S., Natarajan, K., Dotzauer, E., McCallum, I. e Obersteiner, M. (2009) Optimizing biodiesel production in India. Applied Energy, vol. 86, pp. S125-S131.

Liu, S., McDonald, T. e Wang, Y. (2010) Producing biodiesel from high free fatty acids waste cooking oil assisted by radio frequency heating. Fuel, vol. 89, pp. 2735-2740.

Marchetti, J. M. e Errazu, A. F. (2008) Technoeconomic study of supercritical biodiesel production plant. Energy Conversion and Management, vol. 49, pp. 2160-2164.

National Biodiesel Board - NBB (2009) U.S. Biodiesel Production Capacity. Estado Unidos: NBB. Disponível em www.biodiesel.org.

Nuortio, T., Kytojoki, J., Niska, H. e Braysy, O. (2006) Improved route planning and scheduling of waste collection and transport. Expert Systems with Applications, vol. 30, pp. 223-232.

Organization for Economic Co-operation and Development - OECD (2010) Biofuel production 201019. Graph: Global biodiesel production by feedstock. Disponível em www.agri-outlook.org.

Patil, P., Deng, S., Rhodes, J. I. e Lammers, P. J. (2010) Conversion of waste cooking oil to biodiesel using ferric sulfate and supercritical methanol processes. Fuel, vol. 89, pp. 360-364.

Peidong, Z., Yanli, Y., Jin, S., Yonghong, Z., Lisheng, W. e Xinrong, L. (2009) Opportunities and challenges for renewable energy policy in China. Renewable and Sustainable Energy Reviews, vol. 13, pp. 439-449.

Phan, A. N. e Phan, T. M. (2008) Biodiesel production from waste cooking oils. Fuel, vol. 87, pp. 3490-3496. 
Predojevic, Z. J. (2008) The production of biodiesel from waste frying oils: A comparison of different purification steps. Fuel, vol. 87, pp. 3522-3528.

PROVE (2008) Informações a respeito de empresas e de volumes mensais movimentados de óleo residual de fritura na região metropolitana do Rio de Janeiro. Comunicação pessoal, Programa de Reaproveitamento de Óleos Vegetais do Estado do Rio de Janeiro. Rio de Janeiro.

Sakai, T., Kawashima, A. e Koshikawa, T. (2009) Economic assessment of batch biodiesel production processes using homogeneous and heterogeneous alkali catalysts. Bioresource Technology, vol. 100, pp. 3268-3276.

Singh, J., Panesar, B. S. e Sharma, S. K. (2010) A mathematical model for transporting the biomass to biomass based power plant. Biomass and bioenergy, vol. 34, pp. 483-488.

Tarantilis, C. D. (2005) Solving the vehicle routing problem with adaptive memory programming Methodology. Computers \& Operations Research, vol. 32, pp. 2309-2327.

Tavares, G., Zsigraiova, Z., Semiao, V. e Carvalho, M. G. (2009) Optimisation of MSW collection routes for minimum fuel consumption using 3D GIS modeling. Waste Management, vol. 29, pp. 1176-1185.

Transporte Moderno (2008) Custos Operacionais. Revista 431, Ano 45, Brasil.

Vicente, G., Martínez, M. e Aracil, J. (2007) Optimisation of integrated biodiesel production. Part I. A study of the biodiesel purity and yield. Bioresource Technology, vol. 98, pp. 1724-1733.

Wassell Jr., C. S. e Dittmer, T. P. (2006) Are subsidies for biodiesel economically efficient? Energy Policy, vol. 34, pp. 3993-4001.

Wei Li, N., Zong, M. e Wu, Z. (2009) Highly efficient transformation of waste oil to biodiesel by immobilized lipase from Penicillium expansum. Process Biochemistry, vol. 44, pp. 685-688.

Wen, Z., Yu, X., Tu, S., Yan, J. e Dahlquist, E. (2010) Biodiesel production from waste cooking oil catalyzed by TiO2-MgO mixed oxides. Bioresource Technology, vol. 101, pp. 9570-9576.

Zhang, Y., Dubé, M. A., Mclean, D. D. e Kates, M. (2003) Biodiesel production from waste cooking oil: 2. Economic assessment and sensitivity analysis. Bioresource Technology, vol. 90, pp. 229240 . 